\title{
Tribological Anti-Wear and Extreme-Pressure Performance of Multifunctional Metal and Nonmetal Doped C-based Nanodots
}

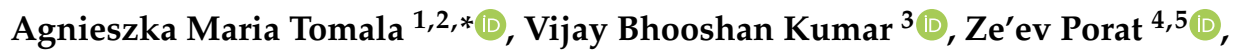 \\ Remigiusz Michalczewski ${ }^{1}$ and Aharon Gedanken ${ }^{2, *}$ \\ 1 Tribology Department, Institute for Sustainable Technologies-National Research Institute (ITeE-PIB), 26-600 \\ Radom, Poland; Remigiusz.Michalczewski@itee.radom.pl \\ $2 \mathrm{AC}^{2} \mathrm{~T}$ research $\mathrm{GmbH}, 2700$ Wiener Neustadt, Austria \\ 3 Bar-Ilan Institute for Nanotechnology and Advanced Materials, Department of Chemistry, Bar-Ilan \\ University, 52900 Ramat-Gan, Israel; vijaybhushan86@gmail.com \\ 4 Division of Chemistry, Nuclear Research Center-Negev, P.O. Box 9001, 84190 Be'er-Sheva, Israel; \\ poratze@post.bgu.ac.il \\ 5 Institutes of Applied Research, Ben-Gurion University of the Negev, 841051 Be'er-Sheva, Israel \\ * Correspondence: agnieszka.tomala@itee.radom.pl (A.M.T.); gedanken@mail.biu.ac.il (A.G.)
}

Received: 7 March 2019; Accepted: 18 April 2019; Published: 23 April 2019

\begin{abstract}
Carbon nanodots (CDs) are extensively explored due to their low toxicity, excellent water solubility and biocompatibility. Particularly, fluorescent CDs have received ever-increasing attention. Nevertheless, only a few works have been published on measuring the tribological properties of doped CDs, especially Ga doped CDs (Ga@CDs) and nitrogen doped CDs (N@CDs), and comparing their tribological properties with those of pristine CDs. In this work we describe a simple one-pot synthesis of CDs and doped CDs, and examine their tribological properties as potential lubricants. It is suggested that doping of CDs with various elements can give them desired properties for anti-wear and extreme-pressure performances.
\end{abstract}

Keywords: Carbon-Dots; metal-doped carbon-dots; tribology

\section{Introduction}

Nanoscale materials is an emerging area of research bearing many potential applications in various fields, such as physical, biological, chemical, clinical, and medical research sciences. Among the advanced nanomaterials in research, carbon materials such as carbon nanotubes (CNTs), graphene, graphene oxide, and graphene quantum dots (GQDs) are cheap and most abundant in our surroundings [1]. In particular, Nanometer Carbon Dots (CDs) have unique physical properties [2-4] which attract much attention for numerous promising applications in industrial nanotechnology. In addition, CDs are environmental-friendly materials and have potential biomedical applications due to their excellent solubility in aqueous medium, negligible photo-bleaching, low cytotoxicity, high biocompatibility, and outstanding photoluminescence [2,5]. The fluorescence quantum yield of CDs can be improved by doping them with metal/nonmetal elements, such as $\mathrm{N}$ and $\mathrm{Ga}$, which have been tested as fluorescent sensors [6]. Moreover, several reports have confirmed that the CDs and N@CDs, if prepared from clean sources, are non-toxic to living organisms, with no significant changes in their vitalities even when used in different concentrations [7-9]. Recently, we have reported a one-step sonochemical approach for making the CDs and Ga@CDs, and utilizing them in multifunctional applications $[3,10,11]$. 
In lubrication science, using nanoscale materials is an emerging concept for enhancement of the tribological performance of machine elements [12]. Present additives include numerous groups or elements (e.g., S, Zn, P, Cl or metals in organic complexes) which release lethal complexes or greenhouse gases upon dilapidation. As a consequence, a number of regulations imply the reduction of $\mathrm{Zn}, \mathrm{S}$, and P content in lubricants [13]. Although it may be impossible to eliminate completely the use of all these elements, it may be feasible to reduce their use by combining them with NPs or compounds with a reduced impact on the environment. Most of the nanomaterials that have been examined include several elements and are more environmentally-friendly [14]. Definitively nanoscale materials in the form of nano-tubes, nano-particles, and nano-dots have the potential to replace toxic additives in future lubrication technologies $[15,16]$.

Regarding fluorescent metal-doped C-dots, only a few reports were published on using CDs as an additive in lubricants to achieve super-lubricity effects but the relevant research is still in infancy [17-20]. CDs exhibited excellent tribological properties since they can increase the load-carrying capacity [21] of a base solution as water-based lubricant additives [22]. Ran et al. also reported a friction coefficient of only 0.009 for a CDs-based polyethylene glycol $(5 / 30 w / w \%)$ composite. This result makes the CDs-composite a better lubrication candidate with respect to pristine CDs or polyethylene glycol solution [23]. There are a few reports on the tribological properties of CDs but none about N@CDs or Ga@CDs. Therefore it is of interest to study the tribological performance of CDs, N@CDs, and Ga@CDs NPs. In particular, doping of CDs could improve their properties as lubricants, for example prolong the lifetime of engine oils and hydraulic oils. Moreover, metal or nonmetal doping of CDs (N@CDs, and Ga@CDs) may also be used as lubricants in artificial joints. The super-lubricity of CDs as additives to a lubricating fluid was reported [18]. Potential applications are worthy of attention regarding the lubrication effect of CDs for different applications.

It is worth mentioning that unlike CDs and N@CDs, for which several synthetic routes exist, the synthesis of Ga@CDs, as well as other low melting point metals as dopants, has been done by the sonochemical method only $[11,16]$.

In this work we report on the facile formation of surfactant-free, catalyst-free CDs, N@CDs, and Ga@CDs. Testing the tribological properties of CDs-based suspensions by ball-on-disc were performed using an $\mathrm{SRV}^{\circledR}$ tribometer.

\section{Experimental Procedures}

\subsection{Chemicals}

Bovine serum albumin (BSA), polyethylene glycol-400 (PEG-400) quinine sulfate and tricaine methanesulfonate (MS222) were purchased from Sigma Aldrich Ltd., Rehovot, Israel. Double-distilled water (DDW, 18.3 MU) was used throughout the experiments.

\subsection{Synthesis and Characterization CDs, Ga@CDs, and N@CDs}

Recently, we reported on the preparation of Ga-doped CDs (Ga@CDs) by ultrasonication method [24]. In brief, a small granule of Ga was inserted into a glass test tube, containing $20 \mathrm{~mL}$ of PEG-400, which was dipped in a water bath at $75{ }^{\circ} \mathrm{C}$. The ultrasonic transducer tip (model VCX 750, frequency $20 \mathrm{kHz}$, voltage $230 \mathrm{~V}$ AC; Sonics and Materials Inc., Newtown, CT, USA) was immersed in the PEG-400 medium, about one inch above the metallic $\mathrm{Ga}$. Once the Ga was melted, ultrasonic irradiation was applied for $3 \mathrm{~h}$ at $70 \%$ amplitude, causing dispersion of the gallium and formation of a light-grey suspension of particles. After the sonication ceased, the solid Ga particles were separated by centrifugation at 12,000 rpm for $30 \mathrm{~min}$. Ga@CDs were found in the light brown-yellow supernatant. CD NPs were prepared by sonication of PEG 400 in a similar manner, without gallium [2].

The synthesis of water-soluble N@CDs was done via a modified hydrothermal method using an aqueous solution of BSA, and its characterization was described previously [25]. Briefly, $250 \mathrm{mg}$ BSA (66.5 kD) were dissolved in $50 \mathrm{~mL}$ of double-distilled water (DDW) and stirred for $30 \mathrm{~min}$ at 
room temperature to prepare a BSA aqueous solution. The resulting solution was transferred into $100 \mathrm{~mL}$ autoclave and heated at $180^{\circ} \mathrm{C}$ for $6 \mathrm{~h}$ in an oven. After completion, the reaction was quenched by cooling the autoclave in water. The carbide slag was discarded from the product solution by centrifugation at 12,000 rpm for $10 \mathrm{~min}$.

The pale yellow-brown suspensions of CDs, N@CDs, and Ga@CDs, were analyzed by several methods. The fluorescence of the aqueous solution of N@CDs was measured by a fluorimeter (Varian Cary Eclipse, Santa Clara, CA, USA). UV-vis analysis of the N@CDs was performed using a Cary 100 spectrophotometer (Varian, Santa Clara, CA, USA), operated by Lab Sphere software. The morphology and crystalline properties of CDs were analyzed by high-resolution transmission electron microscopy (HR-TEM) with JEOL 2100 microscope (Akishima, Tokyo, Japan) at 200 kV. X-ray photoelectron spectroscopy (XPS) and Raman analysis of the CDs materials were performed after drying via lyophilization. XPS analysis of CDs was measured with ESCALAB 250 spectrometer (monochromatic X-ray source of Al Ka excitation (1486.6 eV), Thermo Scientific, Waltham, MA, USA). The dynamic light scattering (DLS) measurements of an aqueous solution of CDs were performed on the ZetaSizer Nano-ZS (Malvern Instruments Ltd., Worcestershire, UK). The Raman spectra of CDs and doped CDs were recorded by a Renishaw using Raman microscope equipped with RL785 and RL830 Class 3B wavelength-stabilized diode lasers and a Leica DM2500 M (Leica Microsystems, Wetzlar, Germany) microscope.

\subsection{Tribological Materials and Methods Selected for Testing CDs, Ga@CDs, and N@CDs}

Tribological tests were performed using a reciprocating ball-on-disc SRV ${ }^{\circledR}$ tribometer (Optimal Instruments Prüftechnik GmbH, Munich, Germany) and four-ball apparatus T-02 according to the ITeE-PIB Polish method for testing lubricants under scuffing conditions [26] (testers are designed and manufactured at ITeE-PIB in Radom). In the SRV method, a $10 \mathrm{~mm}$ diameter ball was loaded and reciprocated against a stationary steel disc under boundary lubricated, pure sliding conditions (test parameters are given in Table 1a). In a T-02 four-ball tester, three stationary bearing steel balls were pressed with increasing load $\mathrm{P}$ against the upper steel ball rotating at a constant speed. The contact zone of the balls was immersed in the tested lubricants (test parameters are given in Table $1 \mathrm{~b}$ ). The T- 02 method was selected as it has been shown to be very sensitive to extreme pressure additives [27].

The base material used for the tribological tests was AISI 52100 bearing steel, with a microstructure formed by fine martensitic iron and disperse micrometer size carbides, which resulted in a hardness of $850 \mathrm{HV} 10$ and a roughness of Ra $0.05 \mu \mathrm{m}$. As the counter body, we also used AISI 52100 bearing steel balls with diameters of $10 \mathrm{~mm}$ (SRV) and $12.7 \mathrm{~mm}$ (T-02) with the same hardness and roughness.

The prepared samples of carbon-based nanodots (CDs), applied as a base lubricant, were compared to the reference NEXBASE ${ }^{\circledR} 2008$ polyalphaolefine (PAO) having viscosity of $7795 \mathrm{~mm}^{2} / \mathrm{s}^{2} 100{ }^{\circ} \mathrm{C}$. The lubricants were subjected to tribological stresses in two manners: with the SRV conditions to determine the anti-wear (AW) properties and with a four-ball tester to evaluate the extreme pressure (EP) properties of the fluids.

After the tribological tests the samples were rinsed with petroleum ether and cleaned in isopropanol in an ultrasonic bath for $3 \mathrm{~min}$ (both solvents were HPLC grade). After cleaning the wear tracks were measured by an optical microscope, an optical interferometer and scanning electron microscope (SEM) with energy dispersive X-ray spectroscopy (EDX). The ball wear scare diameters were measured with a Nikon MM-40/L3FA microscope (Toolmakers Microscopes Stand, Nikon Corporation, Tokyo, Japan) with resolution of $1 \mu \mathrm{m}$. Surface topography of the disc's wear scare was evaluated using a Taylor Hobson CCI HD non-contact 3D Optical Profiler ((Taylor Hobson, San Francisco, CA, USA). Surface roughness was examined before and after the tests according to ISO 4287. The TalyMap Platinum software (Taylor Hobson, San Francisco, CA, USA) was used for wear volume analysis. 
Table 1. Summary of SRV reciprocating sliding tribotest parameters, including a schematic of the experimental set-up.

(b) 4-ball tester

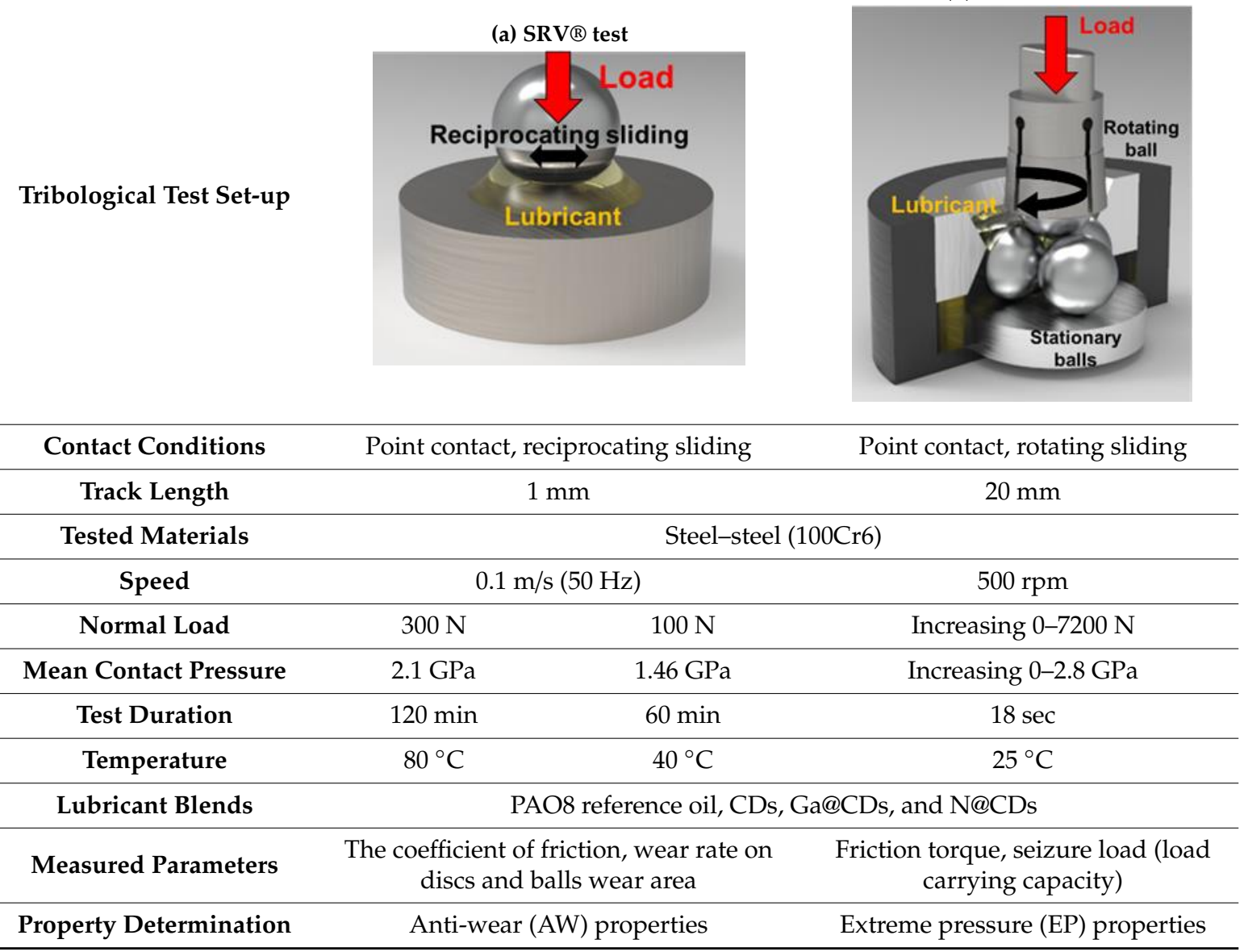

Hitachi SU-70 Analytical Field Emission SEM (Hitachi, Tokyo, Japan) was used to spot the micrographs. It was equipped with the Schottky electron source with ultra-high resolution and EDS (Thermo Scientific). It was operated at magnifications in the range from $30 \times-800,000 \times$ under vacuum of $10^{-8} \mathrm{~Pa}$. The spectra were acquired at an accelerating voltage of $15.0 \mathrm{kV}$ and takeoff angle of 30.9 (distance sample-detector: $15 \mathrm{~mm}$ ).

\section{Results and Discussion}

\subsection{Characterization of CDs, N@CDs and Ga@CDs}

The clear aqueous supernatant suspension of CDs appeared as pale-yellow in daylight (Figure 1a-c) and showed blue-green emission under UV light (Figure 1d-f). The characterization of the CDs [28], N@CDs [25] and Ga@CDs [10,24] have been previously reported and therefore is described here only briefly. The fluorescence of CDs, N@CDs, and Ga@CDs was analyzed at different excitation wavelengths ( $\lambda_{\text {ex }}=330,350,370,390,410,430,450,470$, and $490 \mathrm{~nm}$ ). For pristine CDs and N@CDs, maximum intensities were obtained at $470 \mathrm{~nm}$ for excitation wavelength of $390 \mathrm{~nm}$. Small shifts were observed for Ga@CDs, with maximum fluorescence at $470 \mathrm{~nm}$ for excitation wavelength of $370 \mathrm{~nm}$. (Figure 2a). Other physical characteristic of CDs, N@CDs, and Ga@CDs NPs which were reported in previous works are summarized in Table 2.

TEM images of CDs, N@CDs and Ga@CDs NPs (Figure 3) show that the particles are in the size-range of 4-9 nm. Comparing the images of pristine CDs to those of N@CDs, and Ga@CDs indicates that doping does not affect the particles' size. 

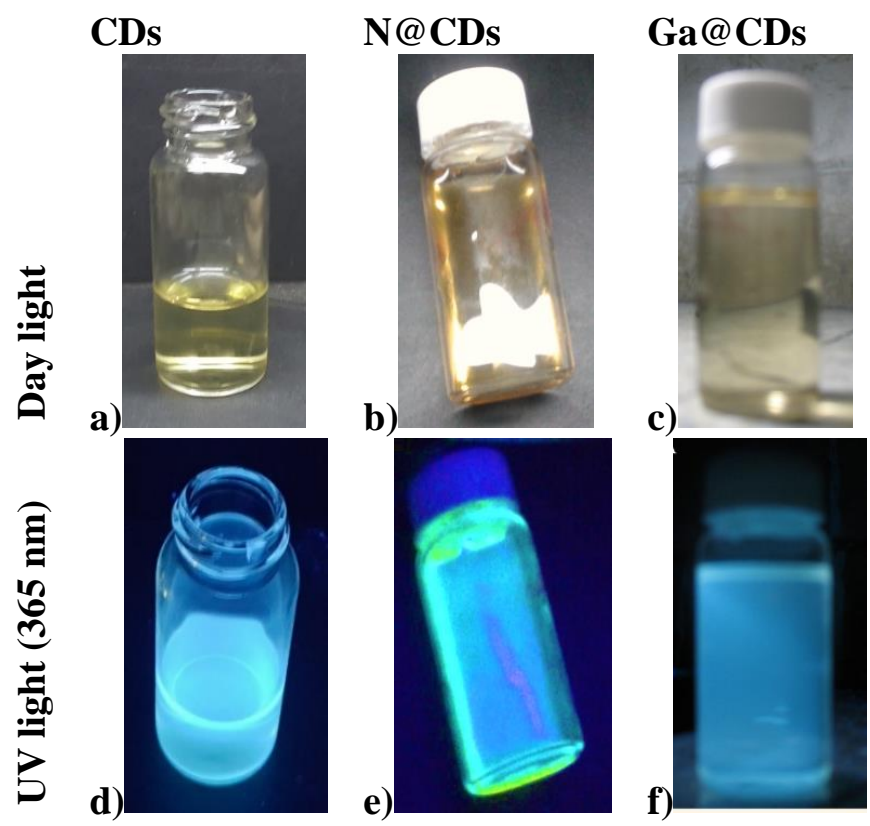

Figure 1. Photographs of CDs, N@CDs, and Ga@CDs suspensions under daylight $(\mathbf{a}, \mathbf{b}, \mathbf{c})$ and UV-light $(\mathbf{d}, \mathbf{e}, \mathbf{f})$.
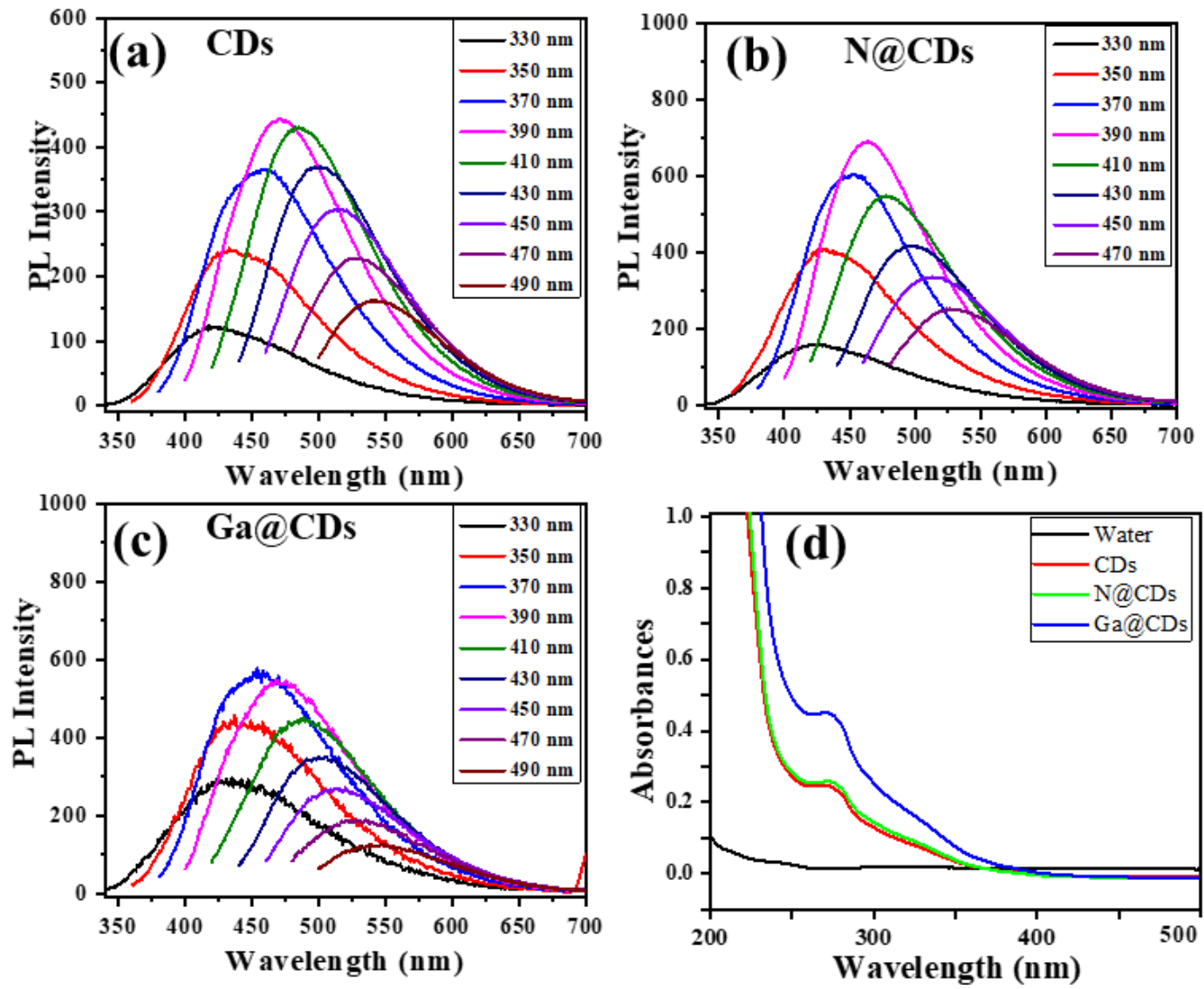

Figure 2. Fluorescence spectra of (a) CDs, (b) N@CDs, and (c) Ga@CDs. (d) Absorption spectra of an aqueous suspension of CDs, N@CDs, Ga@CDs, and water. 
Table 2. Summary of CDs, N@CDs, and Ga@CDs NPs properties. QY: Quantum yield.

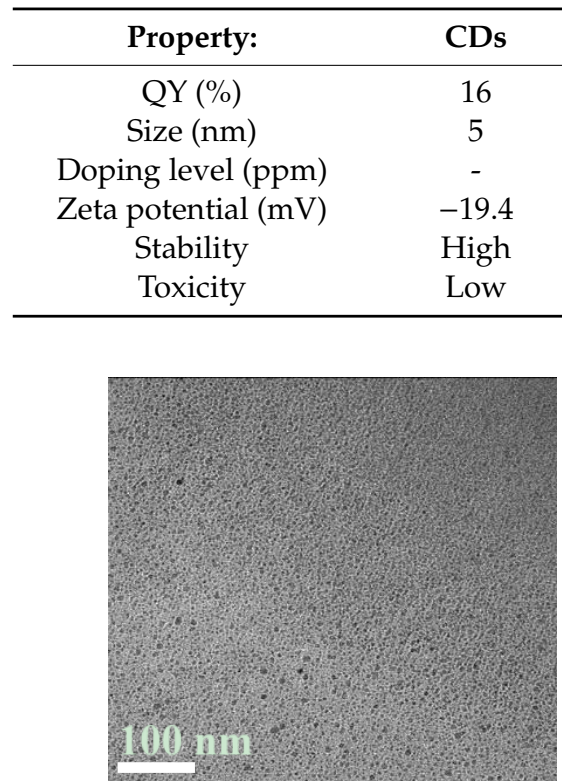

(a)

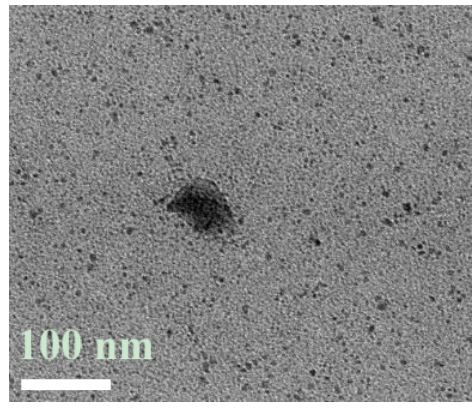

(c)

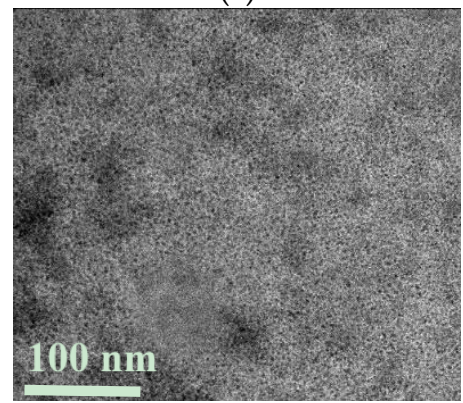

(e)

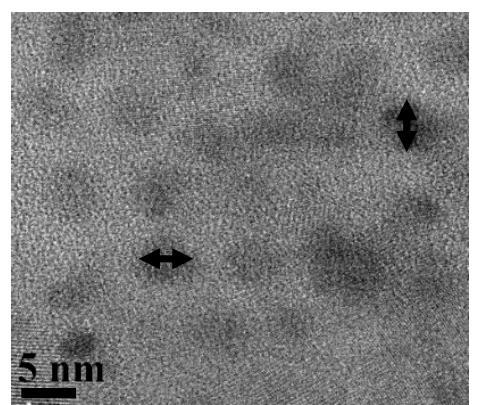

(b)

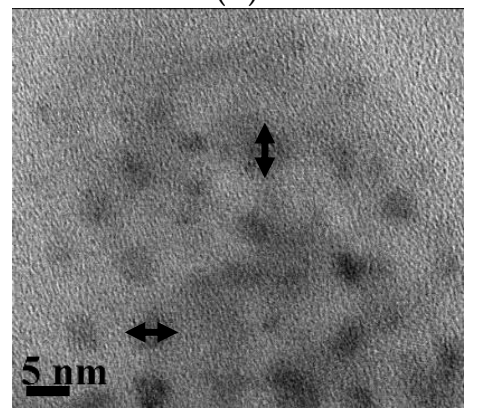

(d)

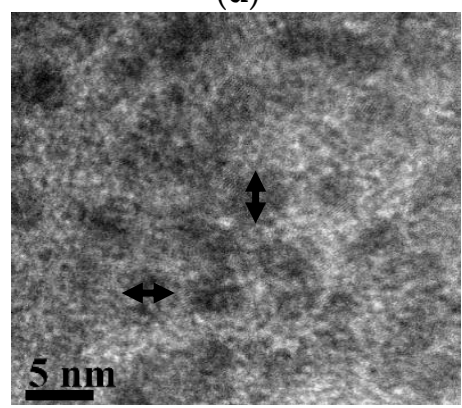

(f)

Figure 3. TEM images of (a,b) CDs, (c,d) N@CDs, and (e,f) Ga@CDs NPs.

Raman spectroscopy of the $\mathrm{N} @$ CDs was used to study the graphitic nature of the carbon. The analysis reveals the presence of several organic functional groups $(-\mathrm{OH},-\mathrm{COOH},-\mathrm{COO},-\mathrm{CO},-\mathrm{O}$ and $-\mathrm{NH}_{2}$ ) on the surface of CDs. These groups enable specific interactions of the with various types of molecules and receptors [29,30].

\subsection{Tribological Performance of CDs, N@CDs, and Ga@CDs}

\subsubsection{Anti-Wear (AW) Properties According to SRV Linear Oscillation Test Method}

The friction coefficients and the anti-wear properties of lubricants based on C-nanodots were measured by reciprocating sliding tests. The tribological performance of the lubricants was evaluated in 
steel-steel material configuration as the most representative tribo-pair in machine elements. Preliminarily parameters were adjusted to follow the ASTM D6425 test method (Table 1a) specified for applications in which high-speed vibrational or start-stop motions are involved, for example, in gear or cam/follower systems. Unfortunately, the test conditions were too harsh due to elevated temperature, particularly for the N@CDs tested fluid. Due to its low viscosity, the fluid evaporated shortly after starting the test (Figure $4 \mathrm{~b}$ ). Therefore, milder test condition were set to fit the properties of the lubricant: $40{ }^{\circ} \mathrm{C}$ temperature, $100 \mathrm{~N}$ load and the test duration of $60 \mathrm{~min}$. The friction curves as a function of time are presented in Figure 4a,b. The error bars on the friction curves are based on three repetitions, showing good reproducibility for all the test conditions. The measured friction coefficient of a reference PAO8 oil was $0.183 \pm 0.008$, in agreement with the reported value [16]. CDs and Ga@CDs were found to have lower friction coefficient than the base oil: $0.129 \pm 0.006$ and $0.132 \pm 0.005$, respectively. These close values can be attributed to the similar surface functional groups, although the compositions of the particles were different. Both CDs and Ga@CDs are based on polyethylene glycol-400 (PEG-400), which has similar viscosity as that of the base oil polyalphaolefine (PAO 8). On the other hand, the N@CDs based lubricant has higher friction coefficient than PAO base oil $(0.216 \pm 0.017$.) It can be assumed that under the harsh testing condition (Figure $4 \mathrm{~b}$ ) comparison between the lubricants was not possible as under milder conditions (Figure 4a). The reasons for this can be the differences in the viscosities of the fluids. N@CDs has low viscosity because it is based on aqueous solution of bovine serum albumin (BSA), which has a final viscosity similar to that of double-distilled water (DDW, 18.3 MU). Under harsh testing conditions (Figure $4 \mathrm{~b}$ ), the N@CDs solution has evaporated from the tribological contact, causing inconsistencies in the measured values of the friction coefficients $(\mathrm{CoF})$. Note the high measured $\mathrm{CoF}$ during the first few seconds of the test for the N@CDs (Figure 4a), probably due to the low viscosity of tested liquid compared to the other fluids and reference PAO oil. Right after the friction SRV test, the wear tracks analysis on the tested disc and balls were performed. Wear volume on the discs was measured and calculated with an optical profilometer, while wear scare on the balls was inspected with an optical microscope. The volume analysis method estimates the volume between the worn surface and a reference plane. The reference plane was set as the average height of the unworn area outside the wear track. The wear volume was calculated for the whole wear track, and only the area under the reference plane was considered. Additionally, the surface height parameters, (kurtosis Sq, skewness Ssk, and roughness Sa) describing the damaged wear track, are given below the figures. The results of the wear zone surface examination are presented in Figure 5. It is clearly visible that the least damaged surfaces, both on the disc and on the ball, are found when the base reference PAO oil was used during the SRV test. The most damaged surface was found for the last lubricant, N@CDs, which is not surprising since the $\mathrm{CoF}$ was also quite high for this lubricant (Figure 4).

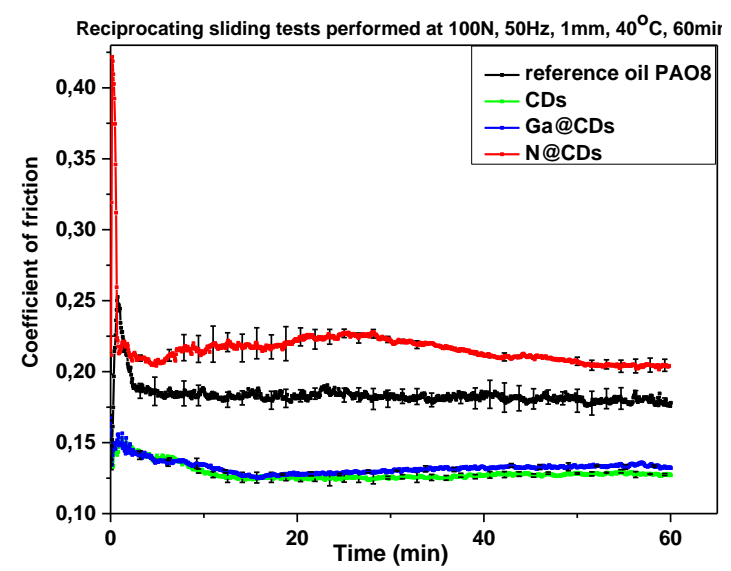

(a)

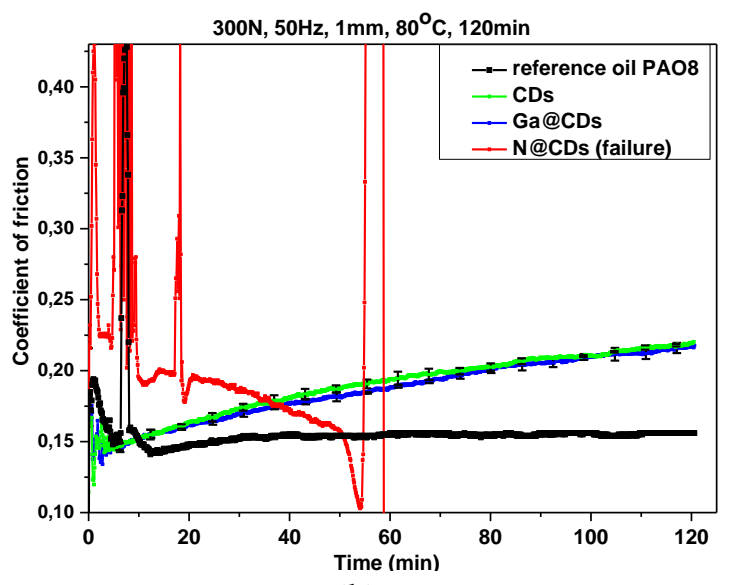

(b)

Figure 4. SRV tests result represented with friction curves including error bars distribution over the curve for the mild test condition (a) and harsh test condition (b). 
The results of the wear tracks' optical analysis are presented in Figure 6, where the discs wear volumes and the balls wear scare diameters are correlated with the tested lubricants. From both Figures 4 and 5 it is evident that C-nanodots do not exhibit AW performance. The reference oil PAO8 has a lower wear rate compared to the tested CDs.

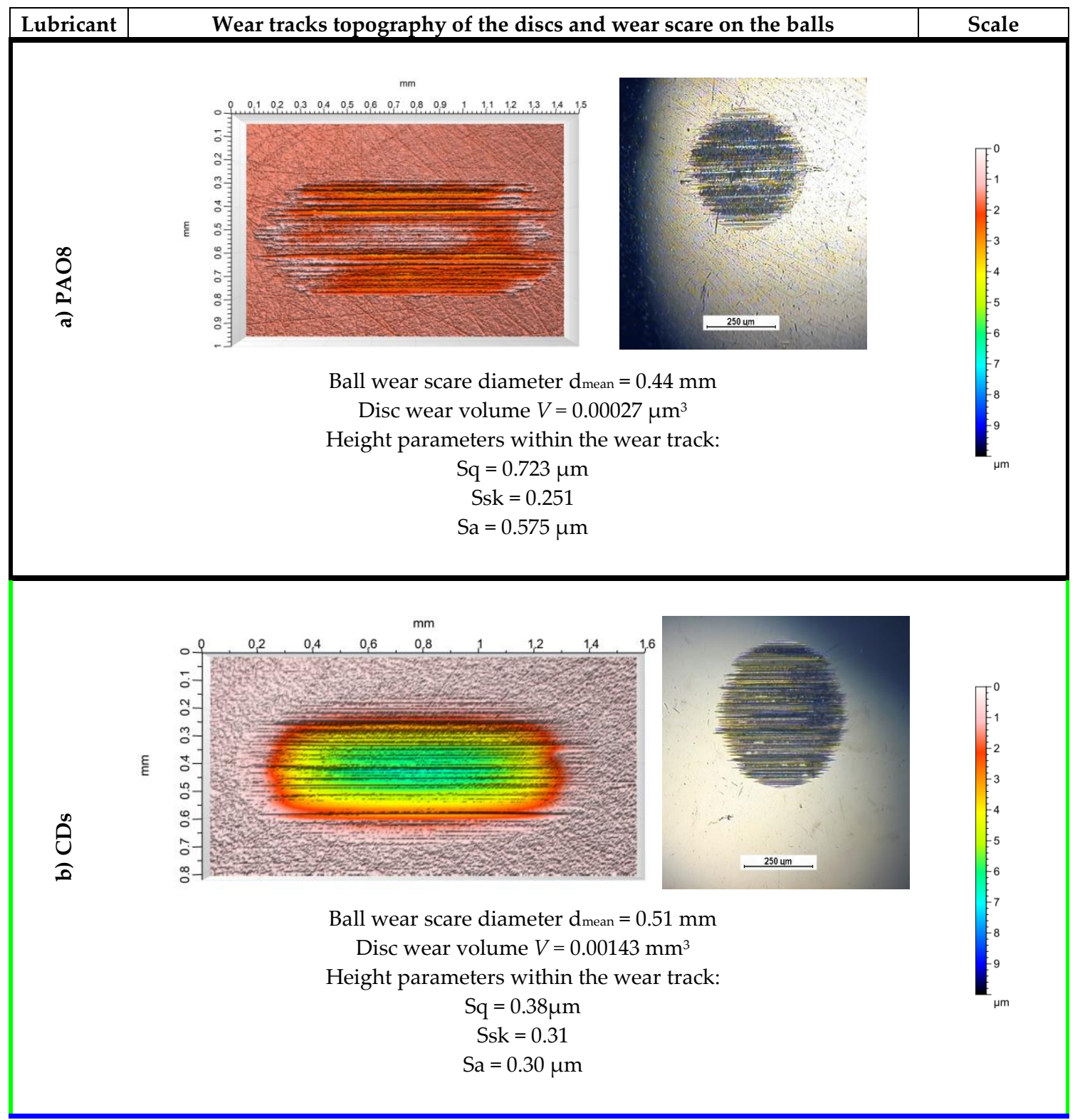

Figure 5. Cont. 


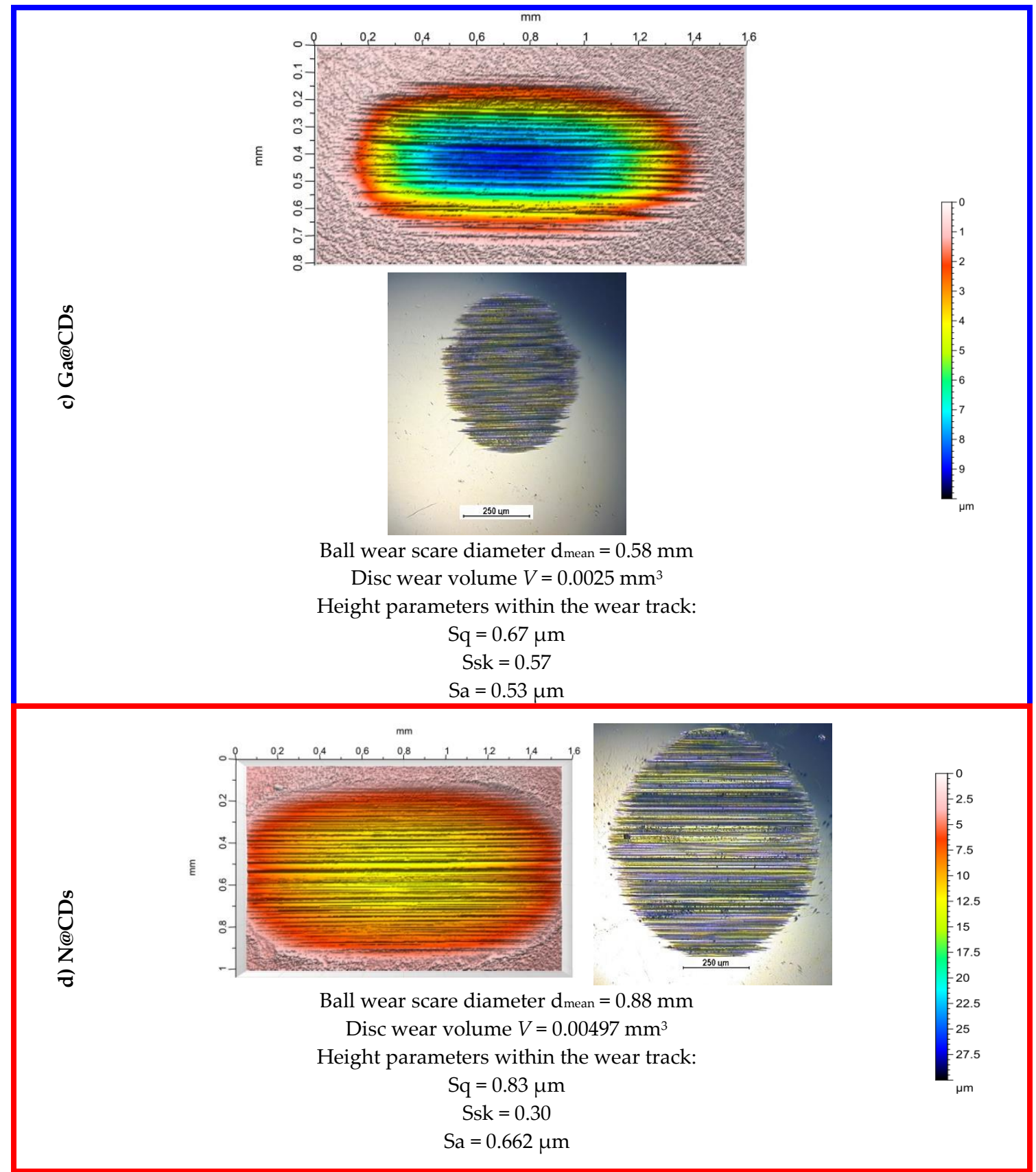

Figure 5. Disc wear track topography with calculated volume and height parameters within the wear track and ball wear scare image, including measured diameter for the following lubricants tested: (a) PAO8, (b) CDs, (c) Ga@CDs, (d) N@CDs. 


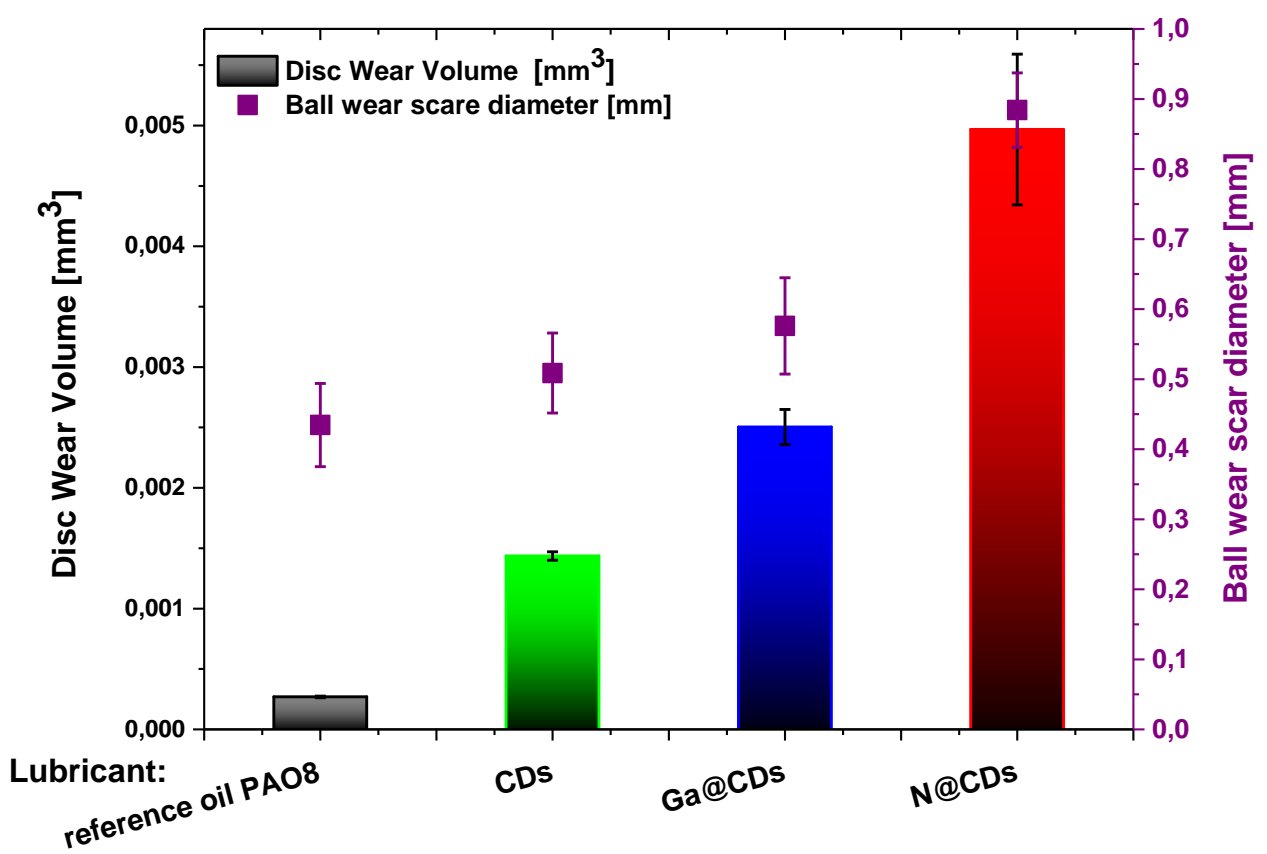

Figure 6. Disc and ball wear of the tested pairs for the different lubricants.

\subsubsection{Extreme Pressure (EP) Properties According to T-02 Four-Ball Tester}

The properties of the tested lubricants related to the prevention of scuffing are called extreme pressure (EP) properties. A unique feature of the four-ball test method is related to the continuous increasing of the load until scuffing and then seizure occur, and to the ability of analyzing the scuffing propagation. During each run the friction torque $M_{t}$ and the applied load are monitored as presented in Figure 7 vs. time. The test run continues until seizure occurs when $10 \mathrm{Nm}$ friction torque is exceeded. The load at this point is called the seizure load, $P_{o z}$. If $10 \mathrm{Nm}$ is not reached (as in the case of the friction torque curve for $\mathrm{N} @ \mathrm{CDs}$ ), the seizure is considered to occur in the end of the run, at the maximum possible load (about 7200N). Surprisingly, for the lubricant which had the highest CoF and wear in SRV AW properties, the test method appears to be the best for performing EP lubricants. For the reference PAO8 oil, as well as for the CDs and Ga@CDs, the seizure load occurred already at $3000 \mathrm{~N}, 3200 \mathrm{~N}$ and $3300 \mathrm{~N}$, respectively. Clearly, there is an extraordinary EP effect of N@CDs on the tribological properties of steel/steel tribosystem; seizure does not occur even at $7200 \mathrm{~N}$. These results were very reproducible. The error bars marked for the N@CDs curve (red color) were calculated for 3 test repetitions and are very narrow for the entire test duration.

For every lubricant which has been tested, the limiting pressure of seizure $\left(p_{o z}\right)$, or in other words the load carrying capacity, has been calculated according to Equation (1) [26]:

$$
p_{o z}=0.52 \frac{P_{o z}}{d^{2}}
$$

where $p_{o z}$ is the limiting pressure of seizure expressed in $\mathrm{N} / \mathrm{mm}^{2}, P_{o z}$ is the seizure load expressed in N, and $d$ is the average wear scare diameter expressed in $\mathrm{mm}$. The higher value of $p_{o z}$ indicates the better extreme-pressure properties of the tested lubricant; that means a higher load carrying capacity. 


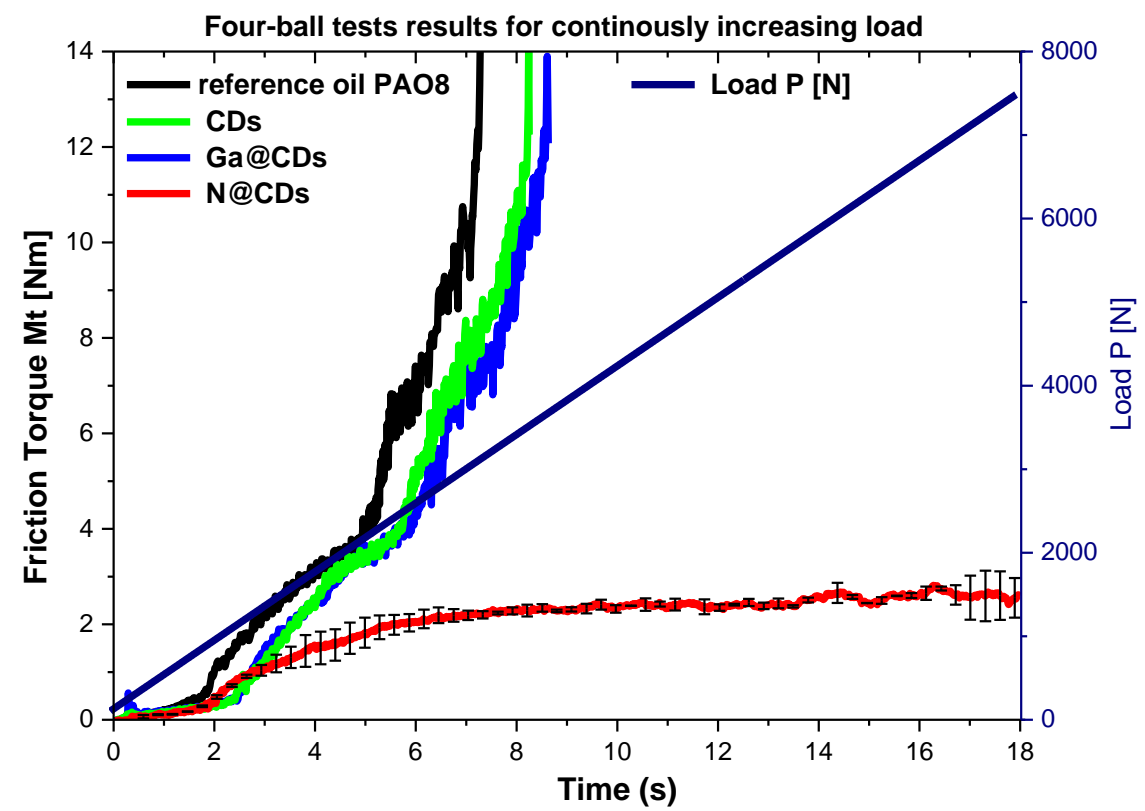

Figure 7. Friction torque curves obtained at the continuously increasing load.

Figure 8 summarizes the calculated scuffing resistance for tested lubricants. It can be seen that the load carrying capacity for N@CDs is higher by an order of magnitude compared to the reference base oil or CDs and Ga@CDs. The application of N@CDs improved the scuffing resistance at least 15 times, and the lubricant provides incredibly stable lubricious film separating steel/steel surfaces from seizure.

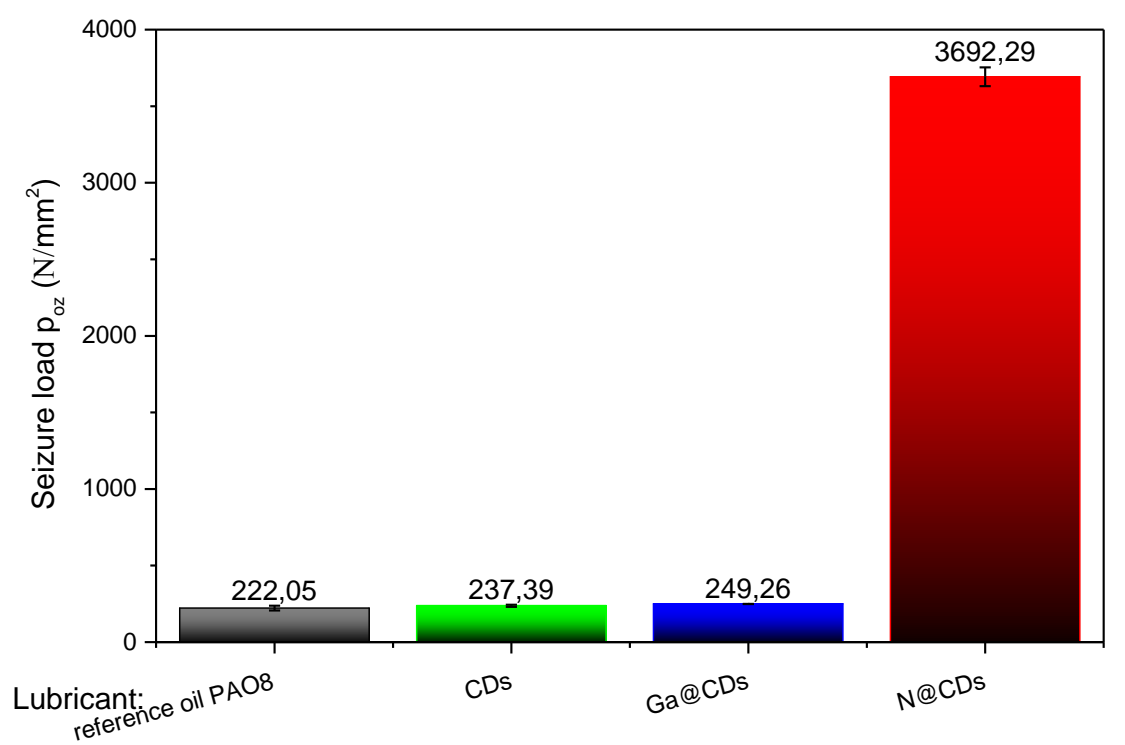

Figure 8. Limiting pressure of seizure $\left(p_{o z}\right)$ obtained for steel/steel tribo-system lubricated with reference PAO8 oil, CDs, Ga@CDs, N@CDs.

In an attempt to understand the wear mechanism for the steel tribo-system lubricated with CDs, SEM/EDX surface analyses were performed on the worn scare after the four-ball tester. The results are presented in Figure 9, showing two SEM images at different magnifications (50 $\times$ and $2000 \times$ ) and the EDX elementary analysis performed at the higher magnification spot for each of the lubricants. Examination of the results shows that the narrowest wear scare appears on the ball after the tests with N@CDs-lubricant. This is in agreement with the observation that this fluid provides the lubricious tribo-film appearing at very low friction torque (Figure 7, red curve) and highest scuffing resistance 
(Figure 8, red column). The N@CDs did not show any instabilities during the test, resulting in extremely narrow wear scare diameter, always smaller than $1 \mathrm{~mm}$ (Figure 9d) while the wear scares for the reference PAO8 oil, CDs and Ga@CDs are larger than $2.5 \mathrm{~mm}$.

The EDX elementary analysis on the worn steel surfaces, after the tribological tests, revealed the presence of the elements $\mathrm{Fe}, \mathrm{Cr}, \mathrm{Mn}, \mathrm{C}$ and traces of $\mathrm{Al}$, originating from the steel substrate. Traces of the elements $\mathrm{C}, \mathrm{Ga}, \mathrm{N}$, and $\mathrm{S}$ were also found, originated from the lubricants. Particularly interesting is the case of the N@CDs lubricant, where $1 \% \mathrm{~S}$ and $1.8 \%$ of $\mathrm{N}$ were detected, which could affect the extremely high EP properties of this lubricant. The source of the sulfur atoms is the BSA molecules which are the precursors for the formation of the N@CDs. In this case, a relatively low concentration of iron and higher concentrations of oxygen and carbon were detected, with respect to the other lubricants, which can be related to the lower friction and tribo-film formation, in agreement with the literature [18].

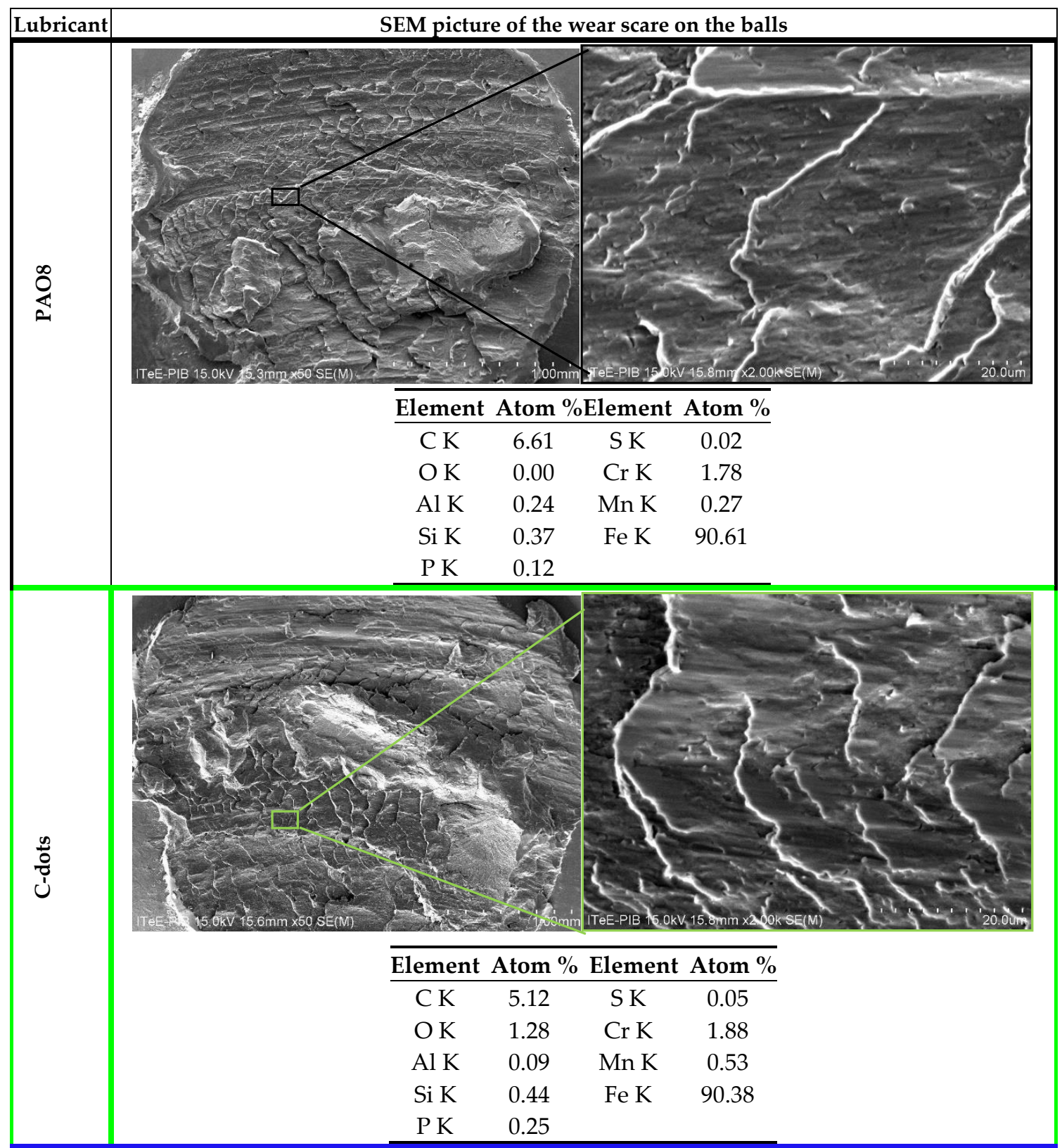

Figure 9. Cont. 


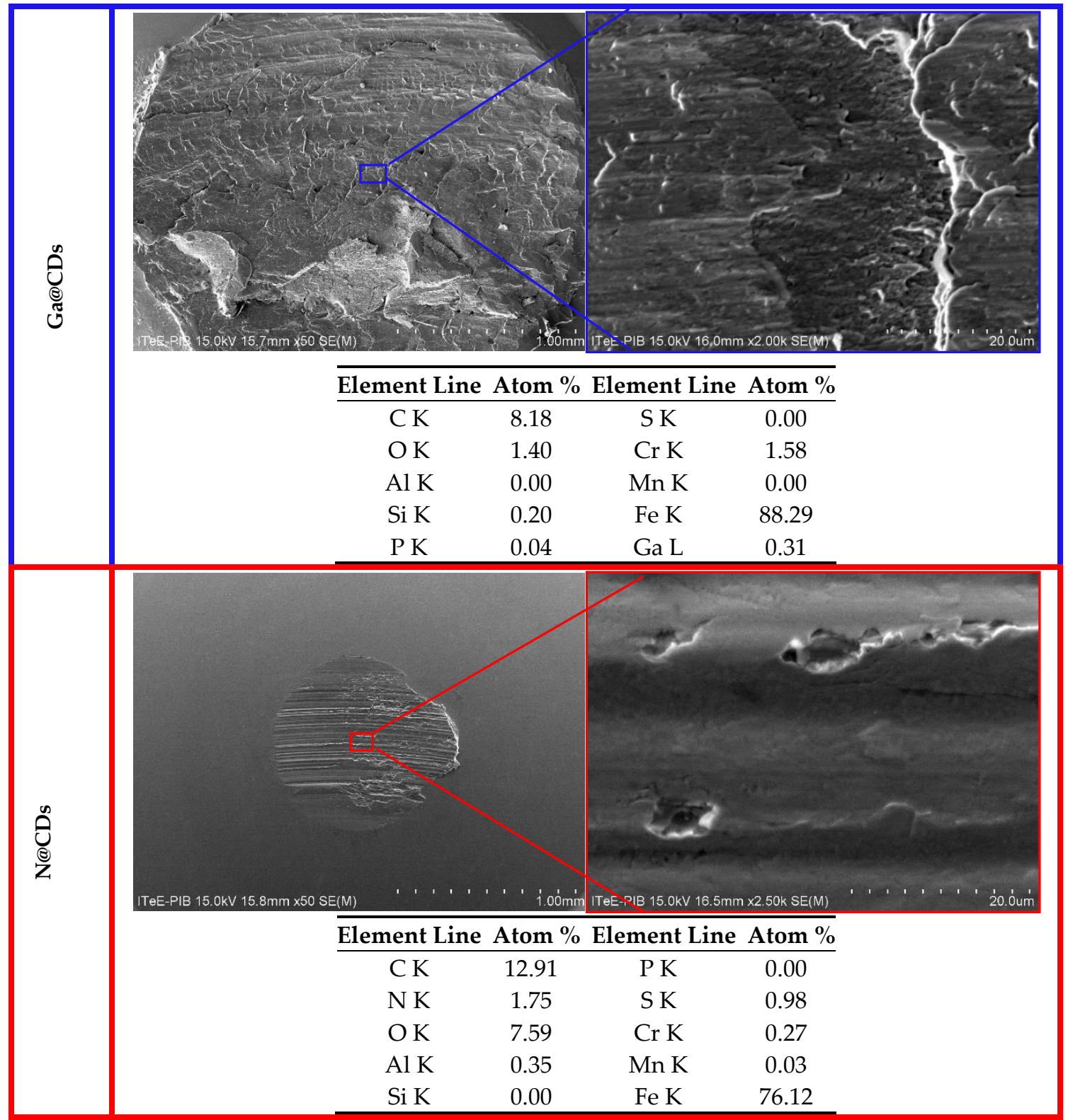

Figure 9. SEM images of the wear scars and EDX elemental composition of the steel surface after the four-ball tests lubricated with (a) reference PAO8 oil, (b) C-dots, (c) Ga@CDs, and (d) N@CDs.

\section{Conclusions}

CDs, N@CDs, and Ga@CDs were prepared by a novel sonochemical method and tested as lubrication materials. Using the SRV reciprocating sliding test method, it was observed that CDs and Ga@CDs have better lubricious properties with respect to N@CDs or even the reference oil. However, the CDs, N@CDs, and Ga@CDs have unsatisfactory anti-wear (AW) activity compared to the reference oil.

Using the four-ball testing machine, the N@CDs lubricant exhibited superb EP performance compared to any other lubricants tested. The good results are comparable to those of the best fully formulated synthetic gear oil with GL-4 class (containing environmental unfriendly additive packages). Doping of CDs with various elements can be used for preparation of lubricants with desired tribological performance, not only for the AW and EP properties but also for tribo-corrosion or cooling. 
Author Contributions: Conceptualization, A.M.T. and A.G.; Methodology, A.M.T., V.B.K. and Z.P.; Software, A.M.T., V.B.K. and Z.P.; Validation, A.M.T., V.B.K. and Z.P.; Formal Analysis, A.M.T., V.B.K. and Z.P.; Investigation, A.M.T., V.B.K. and Z.P.; Resources, R.M. and A.G.; Data Curation, A.M.T., V.B.K. and Z.P.; Writing-Original Draft Preparation, A.M.T., V.B.K., A.G.; Writing-Review \& Editing, all authors; Visualization, A.M.T., V.B.K.; Supervision, R.M. and A.G.; Project Administration, R.M. and A.G.; Funding Acquisition, R.M. and A.G.

Funding: This project has received funding from the European Union's Horizon 2020 research and innovation program under the Marie Skłodowska-Curie grant agreement No 665778. The author, A. M. Tomala, acknowledges the POLONEZ project by National Science Centre, Poland under fellowship registration number 2015/19/P/ST8/02597.

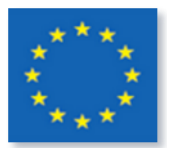

Acknowledgments: The authors are grateful to Ortal Lidor-Shalev of the Department of Chemistry, Bar-Ilan University, Israel, for her help with the TEM measurements. The authors are grateful to Edyta Osuch-Słomka from the Tribology Department of ITeE-PIB for her support with SEM/EDX analyses.

Conflicts of Interest: The authors declare no conflict of interest.

$\begin{array}{ll}\text { Abbreviations } \\ \text { CDs } & \text { carbon-dots } \\ \text { N@CDs } & \text { nitrogen-doped carbon-dots } \\ \text { Ga@CDs } & \text { gallium-doped carbon-dots } \\ \text { BSA } & \text { bovine serum albumin } \\ \text { FWHM } & \text { full-width at half-maximum } \\ \text { HR-TEM } & \text { high-resolution transmission electron microscope } \\ \text { SRV } & \text { linear oscillation test machine } \\ \text { T-02 } & \text { four ball tester } \\ \text { CoF } & \text { coefficient of friction } \\ \text { AW } & \text { anti-wear } \\ \text { EP } & \text { extreme pressure } \\ \text { SEM } & \text { Scanning Electron Microscopy } \\ \text { EDX } & \text { Energy Dispersive X-ray spectroscopy }\end{array}$

\section{References}

1. Jariwala, D.; Sangwan, V.K.; Lauhon, L.J.; Marks, T.J.; Hersam, M.C. Carbon Nanomaterials for Electronics, Optoelectronics, Photovoltaics, and Sensing. Chem. Soc. Rev. 2013, 42, 2824-2860. [CrossRef] [PubMed]

2. Kumar, V.B.; Porat, Z.; Gedanken, A. Facile One-Step Sonochemical Synthesis of Ultrafine and Stable Fluorescent C-Dots. Ultrason. Sonochem. 2016, 28, 367-375. [CrossRef]

3. Kumar, V.B.; Sahu, A.K.; Mohsin, A.S.M.; Li, X.; Gedanken, A. Refractive-Index Tuning of Highly Fluorescent Carbon Dots. ACS Appl. Mater. Interfaces 2017, 9, 28930-28938. [CrossRef] [PubMed]

4. Tangy, A.; Kumar, V.B.; Pulidindi, I.N.; Kinel-Tahan, Y.; Yehoshua, Y.; Gedanken, A. In-Situ Transesterification of Chlorella Vulgaris Using Carbon-Dot Functionalized Strontium Oxide as a Heterogeneous Catalyst under Microwave Irradiation. Energy Fuels 2016, 30, 10602-10610. [CrossRef]

5. Himaja, A.L.; Karthik, P.S.; Singh, S.P. Carbon Dots: The Newest Member of the Carbon Nanomaterials Family. Chem. Rec. 2015, 15, 595-615. [CrossRef] [PubMed]

6. Shi, W.; Li, X.; Ma, H. A Tunable Ratiometric Ph Sensor Based on Carbon Nanodots for the Quantitative Measurement of the Intracellular PH of Whole Cells. Angew. Chem. Int. Ed. 2012, 51, 6432-6435. [CrossRef] [PubMed]

7. Wang, K.; Gao, Z.; Gao, G.; Wo, Y.; Wang, Y.; Shen, G.; Cui, D. Systematic Safety Evaluation on Photoluminescent Carbon Dots. Nanoscale Res. Lett. 2013, 8, 1-9. [CrossRef]

8. Yang, S.T.; Wang, X.; Wang, H.; Lu, F.; Luo, P.G.; Cao, L.; Meziani, M.J.; Liu, J.H.; Liu, Y.; Chen, M.; et al. Carbon Dots as Nontoxic and High-Performance Fluorescence Imaging Agents. J. Phys. Chem. C 2009, 113, 18110-18114. [CrossRef] [PubMed] 
9. Li, S.; Wang, L.; Chusuei, C.C.; Suarez, V.M.; Blackwelder, P.L.; Micic, M.; Orbulescu, J.; Leblanc, R.M. Nontoxic Carbon Dots Potently Inhibit Human Insulin Fibrillation. Chem. Mater. 2015, 27, 1764-1771. [CrossRef]

10. Nissan, I.; Kumar, V.B.; Porat, Z.; Makovec, D.; Shefi, O.; Gedanken, A. Sonochemically-Fabricated Ga@C-Dots@Ga Nanoparticle-Aided Neural Growth. J. Mater. Chem. B 2017, 5, 1371-1379. [CrossRef]

11. Kumar, V.B.; Natan, M.; Jacobi, G.; Porat, Z.; Banin, E. Ga@C-Dots as an Antibacterial Agent for the Eradication of Pseudomonas Aeruginosa. Int. J. Nanomed. 2016, 400, 1-18. [CrossRef]

12. Tomala, A.; Vengudusamy, B.; Rodríguez Ripoll, M.; Naveira Suarez, A.; Remškar, M.; Rosentsveig, R. Interaction Between Selected $\mathrm{MoS}_{2}$ Nanoparticles and ZDDP Tribofilms. Tribol. Lett. 2015, 59, 26. [CrossRef]

13. Naveira-Suarez, A.; Tomala, A.; Pasaribu, R.; Larsson, R.; Gebeshuber, I.C. Evolution of ZDDP-Derived Reaction Layer Morphology with Rubbing Time. Scanning 2010, 32, 294-303. [CrossRef] [PubMed]

14. Tomala, A.; Ripoll, M.R.; Gabler, C.; Remškar, M.; Kalin, M. Interactions between MoS $_{2}$ nanotubes and Conventional Additives in Model Oils. Tribol. Int. 2017, 110, 140-150. [CrossRef]

15. Tomala, A.; Ripoll, M.R.; Kogovšek, J.; Kalin, M.; Bednarska, A.; Michalczewski, R.; Szczerek, M. Synergisms and Antagonisms between $\mathrm{MoS}_{2}$ nanotubes and Representative Oil Additives under Various Contact Conditions. Tribol. Int. 2019, 129, 137-150. [CrossRef]

16. Rodríguez Ripoll, M.; Tomala, A.; Gabler, C.; Dražić, G.; Pirker, L.; Remškar, M. In Situ Tribochemical Sulfurization of Molybdenum Oxide Nanotubes. Nanoscale 2018, 10, 3281-3290. [CrossRef] [PubMed]

17. He, M.; Zhang, J.; Wang, H.; Kong, Y.; Xiao, Y.; Xu, W. Material and Optical Properties of Fluorescent Carbon Quantum Dots Fabricated from Lemon Juice via Hydrothermal Reaction. Nanoscale Res. Lett. 2018, 13, 175. [CrossRef]

18. Ma, W.; Gong, Z.; Gao, K.; Qiang, L.; Zhang, J.; Yu, S. Superlubricity Achieved by Carbon Quantum Dots in Ionic Liquid. Mater. Lett. 2017, 195, 220-223. [CrossRef]

19. Wang, Y.; Hu, A. Carbon Quantum Dots: Synthesis, Properties and Applications. J. Mater. Chem. C 2014, 2, 6921-6939. [CrossRef]

20. Saur, N.; Sanes, J.; Carri, F. Ionanocarbon Lubricants. The Combination of Ionic Liquids and Carbon Nanophases in Tribology. Lubricants 2017, 5, 14.

21. Wang, B.; Tang, W.; Lu, H.; Huang, Z. Ionic Liquid Capped Carbon Dots as a High-Performance Friction-Reducing and Antiwear Additive for Poly(Ethylene Glycol). J. Mater. Chem. A 2016, 4, 7257-7265. [CrossRef]

22. Liu, X.; Huang, Z.; Tang, W.; Wang, B. Remarkable Lubricating Effect of Ionic Liquid Modified Carbon Dots as a Kind of Water-Based Lubricant Additives. Nano 2017, 12, 1750108. [CrossRef]

23. Lu, H.; Ren, S.; Zhang, P.; Guo, J.; Li, J.; Dong, G. Laser-Textured Surface Storing a Carbon Dots/Poly(Ethylene Glycol)/Chitosan Gel with Slow-Release Lubrication Effect. RSC Adv. 2017, 7, 21600-21606. [CrossRef]

24. Kumar, V.B.; Perelshtein, I.; Lipovsky, A.; Porat, Z.; Gedanken, A. The Sonochemical Synthesis of Ga@C-Dots Particles. RSC Adv. 2015, 5, 25533-25540. [CrossRef]

25. Kumar, V.B.; Sheinberger, J.; Porat, Z.; Shav-Tal, Y.; Gedanken, A. A Hydrothermal Reaction of an Aqueous Solution of BSA Yields Highly Fluorescent N Doped C-Dots Used for Imaging of Live Mammalian Cells. J. Mater. Chem. B 2016, 4, 2913-2920. [CrossRef]

26. Piekoszewski, W.; Szczerek, M.; Tuszynski, W. The Action of Lubricants under Extreme Pressure Conditions in a Modified. Wear 2001, 249, 188-193. [CrossRef]

27. Peña-Parás, L.; Taha-Tijerina, J.; Garza, L.; Maldonado-Cortés, D.; Michalczewski, R.; Lapray, C. Effect of $\mathrm{CuO}$ and $\mathrm{Al}_{2} \mathrm{O}_{3}$ Nanoparticle Additives on the Tribological Behavior of Fully Formulated Oils. Wear 2015, 332-333, 1256-1261. [CrossRef]

28. Kumar, V.B.; Dolitzky, A.; Michaeli, S.; Gedanken, A. Antiparasitic Ointment Based on a Biocompatibile Carbon Dot Nanocomposite. ACS Appl. Nano. Mater. 2018, 1, 1784-1791. [CrossRef]

29. Hong, G.; Diao, S.; Antaris, A.L.; Dai, H. Carbon Nanomaterials for Biological Imaging and Nanomedicinal Therapy. Chem. Rev. 2015, 115, 10816-10906. [CrossRef]

30. Wang, L.; Yin, Y.; Jain, A.; Zhou, H.S. Aqueous Phase Synthesis of Highly Luminescent, Nitrogen-Doped Carbon Dots and Their Application as Bioimaging Agents. Langmuir 2014, 30, 14270-14275. [CrossRef] 